\title{
Whole-body imaging of the musculoskeletal system: the value of MR imaging
}

\author{
Gerwin P. Schmidt • Maximilian F. Reiser • \\ Andrea Baur-Melnyk
}

Received: 17 December 2006 /Revised: 25 March 2007 / Accepted: 9 April 2007 / Published online: 7 June 2007

(C) ISS 2007

\begin{abstract}
In clinical practice various modalities are used for whole-body imaging of the musculoskeletal system, including radiography, bone scintigraphy, computed tomography, magnetic resonance imaging (MRI), and positron emission tomography-computed tomography (PET-CT). Multislice CT is far more sensitive than radiographs in the assessment of trabecular and cortical bone destruction and allows for evaluation of fracture risk. The introduction of combined PET-CT scanners has markedly increased diagnostic accuracy for the detection of skeletal metastases compared with PET alone. The unique soft-tissue contrast of MRI enables for precise assessment of bone marrow infiltration and adjacent soft tissue structures so that alterations within the bone marrow may be detected before osseous destruction becomes apparent in CT or metabolic changes occur on bone scintigraphy or PET scan. Improvements in hard- and software, including parallel image acquisition acceleration, have made high resolution wholebody MRI clinically feasible. Whole-body MRI has successfully been applied for bone marrow screening of metastasis and systemic primary bone malignancies, like multiple myeloma. Furthermore, it has recently been proposed for the assessment of systemic bone diseases predisposing for malignancy (e.g., multiple cartilaginous exostoses) and muscle disease (e.g., muscle dystrophy). The following article gives an overview on state-of-the-art whole-body imaging of the musculoskeletal system and highlights present and potential future applications, especially in the field of whole-body MRI.
\end{abstract}

\footnotetext{
G. P. Schmidt $(\square) \cdot$ M. F. Reiser $\cdot$ A. Baur-Melnyk Institute of Clinical Radiology,

University Hospitals Munich/Grosshadern, LMU,

Marchioninistrasse 15,

81377 Munich, Germany

e-mail: Gerwin.Schmidt@med.uni-muenchen.de
}

Keywords Whole-body · Imaging · Musculoskeletal · Magnetic resonance imaging

\section{Introduction}

The skeletal system is a frequent target of metastatic spread from various primary tumors like carcinoma of the breast, lung and prostate cancer. Moreover, primary malignancies may also originate from the bone marrow, such as lymphoma and multiple myeloma [1]. Therefore, it is highly important to accurately assess manifestations of malignant diseases within the bone marrow in order to facilitate adequate therapy and predict prognosis.

Only pronounced destruction of bone with loss of bone mineral content exceeding $50 \%$ is readily visible in radiographic examinations [2]. Computed tomography (CT) is definitely more sensitive than radiography and it is the image modality of choice to evaluate the extent of destruction of trabecular and cortical bone and to assess stability and fracture risk. Magnetic resonance imaging (MRI), on the other hand, allows bone marrow components, such as hematopoietic and fat cells, to be visualized. Moreover, tumor infiltration into the spinal canal and paravertebral soft tissues is clearly depicted. Compared with other imaging modalities like radiography, CT or bone scintigraphy, it is the most sensitive technique for the detection of pathologies restricted to the bone marrow, even if trabecular bone is not destroyed $[3,4]$. It has been reported that up to $40 \%$ of skeletal metastases occur outside the field of view covered by a routine assessment of the axial skeleton, underlining the importance of whole-body bone marrow imaging [5]. In the past, different requirements for patient positioning and coil set-up complicated the introduction of MRI as a practicable whole-body application. With multi-channel whole-body MRI 
(WB-MRI) scanners, however, head-to-toe assessment of the whole skeletal system has become a realistic option without compromises in image quality compared with dedicated examinations of limited anatomical areas. Beyond the assessment of malignant bone neoplasms, WB-MRI has recently been proposed for the whole-body imaging of systemic muscle disease and may prove useful for an evidence-based screening of patients suffering from diseases that predispose to bone malignancy (e.g., multiple cartilaginous exostoses).

\section{Technical aspects of whole-body MRI}

Due to its lack of ionizing radiation MRI seems suitable for whole-body imaging, but for a long time its primary application has been the assessment of focal pathologies within particular organs and body parts. The most severe challenges of WB-MRI in the past have been long examination times, mainly caused by time-consuming patient repositioning and changing of the array configuration. Initially, the sequential scanning approach for WB-MRI of the skeletal system consisted of separate scanning steps of T1-weighted and STIR (short tau inversion recovery) imaging at five body levels with at least one patient repositioning process using conventional head, neck, body, and spine array coils. For a complete whole-body examination, including dedicated imaging of the spine in sagittal orientation, a total room time of at least $60 \mathrm{~min}$ had to be taken in account. Steinborn et al. introduced for the first time this whole-body bone marrow scanning concept for the screening of bone metastases and, despite the considerable complexity of the examination, reported advantages in diagnostic accuracy for MRI compared with conventional WB imaging techniques, like skeletal scintigraphy [6]. Later, attempts to overcome FOV restrictions and increase patient comfort were based on a rolling platform concept mounted on top of the scanner table, making the patient glide in between a "coil sandwich" comprised of the body coil and the integrated spine coil (AngioSURFTM/BodySURFTM; MR-Innovation, Essen, Germany). However, with this approach considerable compromises in spatial resolution, especially in peripheral body regions like the head/neck and lower extremities, had to be tolerated [7]. With the introduction of multi-channel MR scanners, using a system of multiple phased array coils covering the whole body like a matrix, imaging of the total skeletal system without compromises in spatial resolution became possible. In particular, the combination of free table movement with parallel imaging acquisition techniques (PAT) resulted in substantially shorter room time and allowed to integrate otherwise time-consuming, but indispensable sequence types for bone marrow imaging (e.g., STIR sequences). The proposed imaging protocol for high resolution T1-weighted turbo spin echo (TSE) and STIR imaging from head to toe is performed with $1.3 \times 1.1-\mathrm{mm}$ and $1.8 \times 1.3-\mathrm{mm}$ plane resolution respectively (5-mm slices, matrix 384 , PAT 2-3). Additionally, dedicated T1-weighted TSE and STIR imaging of the complete spine is performed $(1.0 \times 1.0-\mathrm{mm}$ plane, 3-mm slices, matrix 384), which results in a total scan time of $43 \mathrm{~min}$.

A promising new concept for WB-MRI is the continuously moving table technique with the use of PAT. Recently, a SENSE reconstruction algorithm has been successfully applied on stationary receiver coils with arbitrary coil dimensions for continuously 3D gradient echo imaging of the complete body without significant constraints in image quality [8]. Zenge et al. have reported promising initial results for 3D whole-body continuous data acquisition using the rolling table concept as a new potential strategy for WB-MRI metastases screening, especially for large field-of-view imaging in short bore systems [9].

\section{Technical aspects of whole-body CT}

Multislice CT (MS-CT) is frequently used in oncologic imaging, and in the detection of bone destruction CT is far more sensitive than radiography [10]. Moreover, it is unique in its ability to evaluate the extent of osseous destruction and to assess stability and fracture risk [11]. Whole-body imaging usually implies CT of the neck, thorax, abdomen/pelvis in axial orientation with multi-planar reconstructions and recalculation of bone window setting. Continuous hardware improvements for $\mathrm{CT}$ imaging from single-slice scanners to 64-slice scanners have resulted in larger FOV and faster acquisition times with high resolution. Examination time now is reduced to 1-2 min and improvement in resolution with the resulting formation of isotropic voxels allows high quality multiplanar reconstructions. In particular, the use of ultrathin collimation $(0,5 \mathrm{~mm})$ can provide excellent image quality for the neck and peripheral skeleton. Furthermore, with automated tube current dose modulation systems exposure of the patient to ionizing radiation could be reduced by $10-68 \%$ on average, depending on the anatomical region, without substantially sacrificing image quality [12]. Absolute dose reduction (described as volume CT dose index) ranges from 11.97/ $8.18 \mathrm{mGy}$ for a pelvic examination to $23.28 / 7.45 \mathrm{mGy}$ when a combined angular and z-axis modulation system is used.

Multislice CT enables bone destruction to be detected as well as osteoblastic and mixed patterns with osteosclerosis and destruction. In cases of diffuse bone marrow infiltration inhomogeneous osteoporosis may be detected. However, as in radiography, this may mimic osteoporosis, unless areas of bony destruction are present. 3D reconstruction algorithms, like VRT (volume rendering technique), can be used to display complex fractures (e.g., pathological fracture) and further increases the reliability to detect occult vertebral fractures compared with plain radiographs. Furthermore, the 
3D morphology of vertebral fractures may give indications concerning the nature of the fracture (e.g., tumorous vs. osteoporotic fracture).

\section{Clinical application of whole-body MRI}

Whole-body MRI is increasingly used in the field of oncologic imaging as an adjunct or alternative to established multi-modality approaches (e.g., radiographs, MS-CT, ultrasound, scintigraphy) for initial tumor staging or screening for tumor recurrence after curative therapy. Promising results have been reported for the detection of distant metastatic disease, especially in tumors that frequently metastasize to the bone, liver, and brain (Fig. 1) [13, 31]. Recently, WBMRI has been proposed as a sensible application for a more integrated assessment of multiple myeloma and systemic muscle diseases [16, 17, 18, 19].

\section{Metastasis}

Based on morphological criteria in radiography, CT, and MRI, skeletal metastases are classified as osteolytic (approximately $50 \%$ ), osteoblastic (35\%) , and mixed type (15\%). For MRI bone screening, the combination of unenhanced T1-weighted spin echo and turbo-STIR sequences proved to be highly sensitive in discriminating benign from malignant marrow disorders [20]. On T1-weighted sequences tumor spread is identified by replacement of normal fat containing marrow, resulting in a hypointense signal . Fat-suppressed sequences, such as STIR, depict neoplastic lesions by virtue of the hyperintense signal due to increased content of water within the tumor cells (Fig. 2). However, osteoblastic metastases may be depicted in STIR sequences with variable signal intensities from hypointense in dense sclerotic lesions to hyperintense when more cellular components are present [21]. The unique soft-tissue contrast of MRI allows precise assessment of tumor infiltration within the bone marrow and even diffuse infiltration of the bone marrow with neoplastic cells, not associated with focal bone destructions or formation of new bone, is detected [22]. In some cases additional sequences, such as dynamic studies of signal enhancement after gadolinium injection, may be performed for accurate differentiation from benign bone marrow changes, such as hyperplastic bone marrow formation [23]. Finally, MRI allows for precise assessment of adjacent paraosseous structures, such as the spinal canal.

\section{Whole-body MRI compared with bone scintigraphy}

In clinical practice multi-modality algorithms are most commonly used when metastatic bone disease is suspected. They include radiography, bone scintigraphy, PET, CT, and MRI. In many institutions ${ }^{99 \mathrm{~m}} \mathrm{Tc}$-phosphonate-based bone scanning is performed as the method of initial bone marrow screening. However, scintigraphy provides only limited spatial resolution and at an early stage of disease lesions may remain invisible in the absence of an osteoblastic response [24]. Furthermore, misinterpretation of tracer uptake in healing fractures or degenerative disease may lead to false-positive findings. The diagnostic performance of MRI compared with bone scintigraphy for the detection of skeletal metastases has been examined in various studies and higher specificity and sensitivity in the early detection of skeletal metastases have been reported [6, 24, 25]. Steinborn et al. compared combined T1-weighted and STIR WB-MRI with bone scintigraphy using the sequential scanning approach in a lesion-by-lesion analysis [6]. WB-MRI reliably detected more confirmed skeletal metastases (91\%) than bone scintigraphy (85\%). Another study group reported higher sensitivity and specificity of WB-MRI $(92 \% / 90 \%)$ compared with scintigraphy $(83 \%$ / $80 \%$ ) on a patient-by-patient basis [20]. Daldrup-Link et al. analyzed both methods for the detection of bone metastases in 39 children and young adults and observed a higher sensitivity of $82 \%$ for WB-MRI compared with $71 \%$ for scintigraphy, with this difference increasing, especially in medium-sized lesions between 1 and $5 \mathrm{~cm}$ [3].

Interestingly, both methods showed differences in performance according to lesion location. Most false-negatives in scintigraphy were found in the spine, while diagnostic problems for WB-MRI occur in the thoracic cage and skull, especially when coronal imaging orientation is used, a problem that is certainly increased by motion artefacts. These problems might be overcome when using fast turbo spin echo sequences for thoracic imaging in combination with axial slice orientation.

\section{Whole-body MRI compared with MS-CT}

Multislice CT is far more sensitive than radiography in the assessment of focal bone destructions. Krahe et al. compared radiography and $\mathrm{CT}$ examinations in 112 patients with metastases of the spine [10]. MS-CT identified 268 involved vertebrae while radiography depicted $88 \%$ of these lesions when the vertebral body was involved and only $66 \%$ when other parts of the vertebrae were affected. Intraspinal and paravertebral tumor extension was correctly assessed by plain radiography in only $23 \%$ and $33 \%$ of cases respectively. Recently, multidetector MS-CT has been proposed for whole-body screening of the skeletal system as an alternative to bone scintigraphy [26]. Groves et al. investigated 43 patients with suspected bone metastases using bone scintigraphy and 16-detector MS-CT and detected metastatic disease in 14 out of 43 and 13 out of 43 patients respectively, with an agreement of $84 \%$, suggesting a similar diagnostic performance of both modalities. The authors concluded that in case of suspected skeletal metastasis, scintigraphic studies 

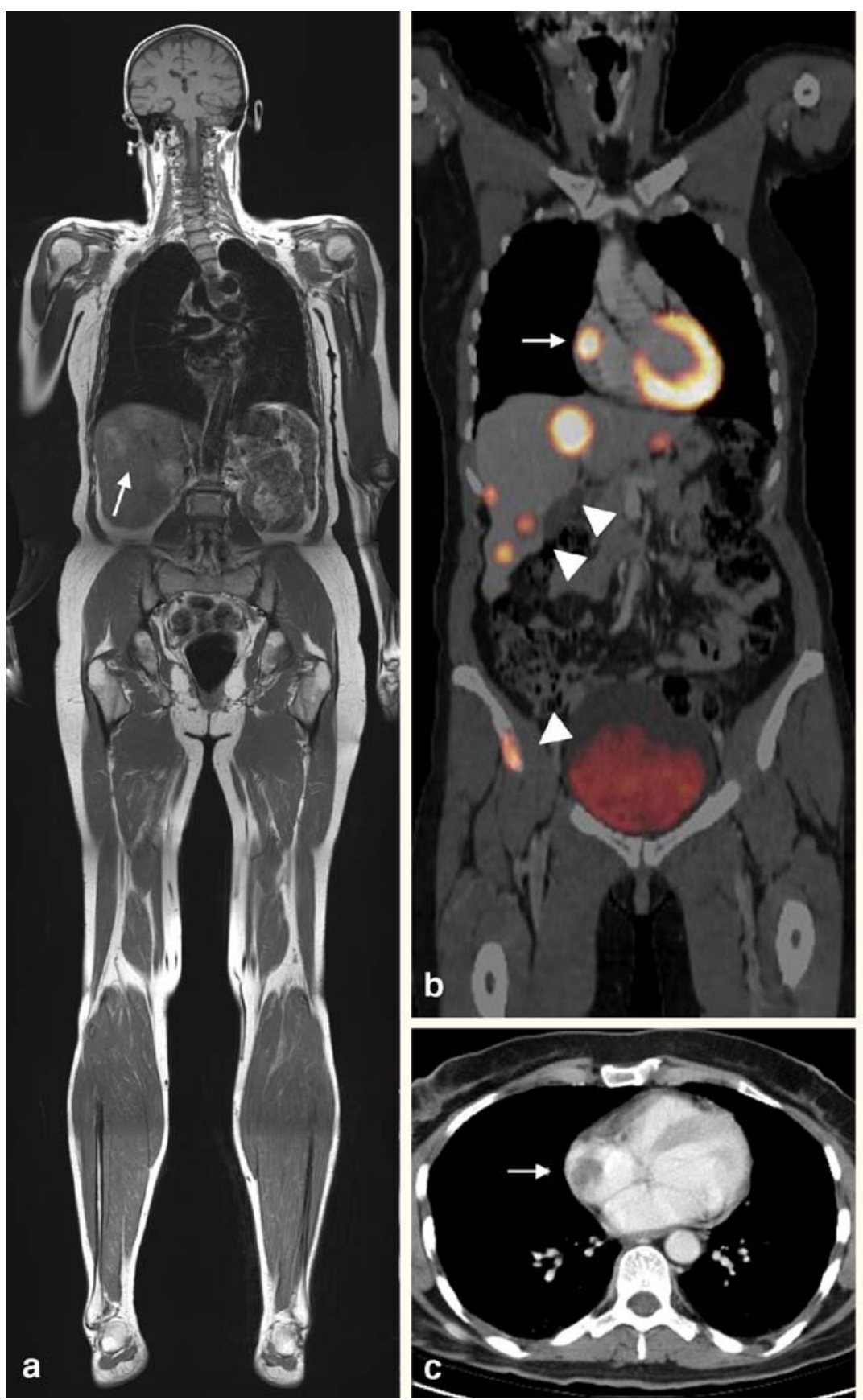

Fig. 1 A 60-year-old patient with a malignant melanoma. a Coronal T1-weighted turbo spin echo (TSE) whole-body (WB) magnetic resonance imaging (MRI) indicates multifocal metastatic disease of the liver (arrow). b Whole-body positron emission tomographycomputed tomography (PET-CT) shows multiple areas of pathologic [18F]-fluorodeoxyglucose (FDG)-uptake in the right atrium (arrow), the liver (arrowheads), and in the right iliac bone (arrowhead). c

may not be needed, when an adequate whole-body MS-CT tumor staging with recalculation of bone window setting has been performed. Consecutively, this might shorten diagnostic pathways and save resources. However, it is not yet clear whether MS-CT is equal to MRI for the assessment of bony metastases. First results have demonstrated the superior detection rate of MRI (Fig. 1) [27].
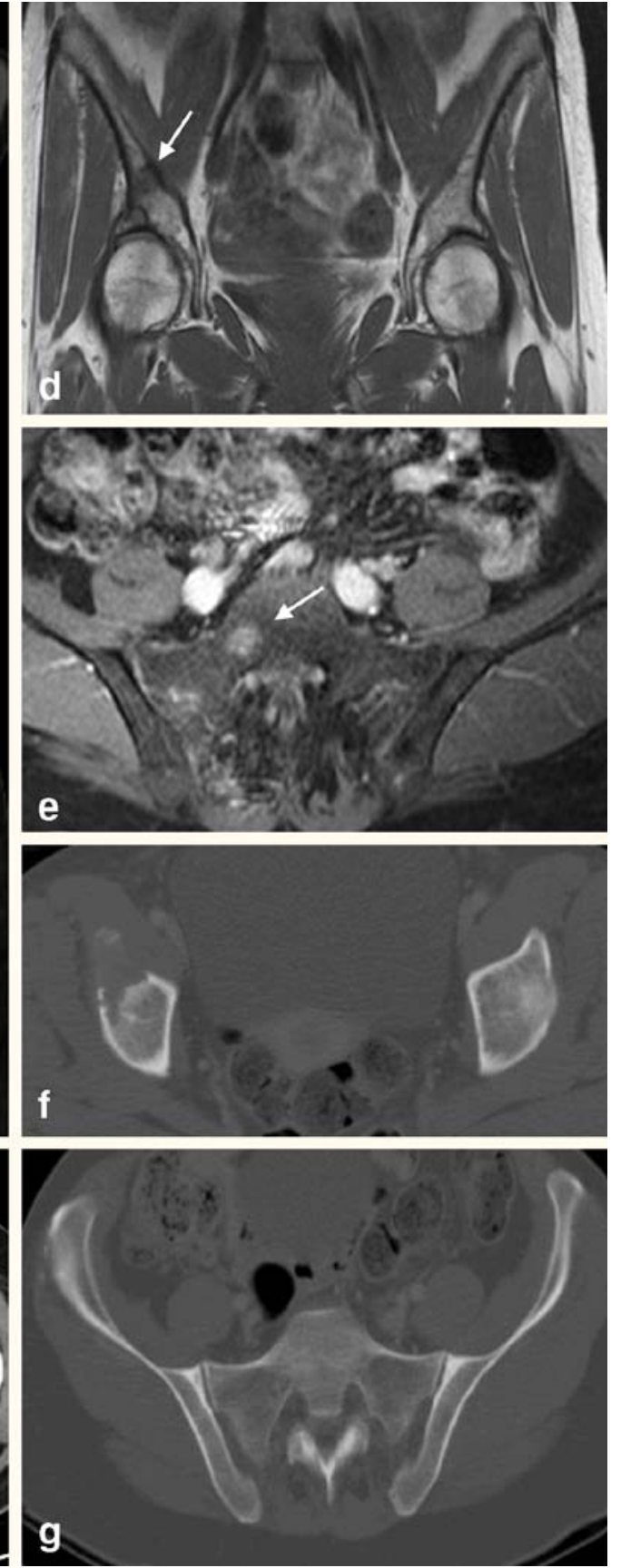
T1-weighted TSE WB-MRI confirms a hypointense lesion in the right iliac bone indicating bone metastasis. e Axial fat-saturated contrast-enhanced MRI of the pelvis shows another bone metastasis in the right sacral bone (arrow). f, $\mathbf{g}$ The corresponding CT images in the bone window setting show extensive osteolysis within the right iliac bone; however, no morphologic changes are found in the right sacral bone

\section{Whole-body MRI compared with PET-CT}

In contrast to MRI and MS-CT, positron emission tomography (PET) using [18F]-fluorodeoxyglucose (FDG) provides functional information by tracing increased FDG uptake directly into the tumor cells. Recent studies indicate that whole-body FDG-PET increases specificity of bone 
screening compared with bone scintigraphy, although there is conflicting evidence whether there is a significant gain in sensitivity [28]. Still, FDG is a tracer that is not tumorspecific and may also accumulate in the presence of inflammation and thus lead to false-positive findings. Also, FDG is not suitable for several tumor entities due to poor tracer uptake, e.g., prostate cancer, myxoid tumors of the gastrointestinal tract, low-grade sarcomas or renal cell carcinomas [29]. A clear technical disadvantage of PET, similar to scintigraphy, is its poor anatomical resolution, which often makes the exact localization of a lesion difficult. Fused PET-CT scanners combine the functional data of PET with the detailed anatomical information of MS-CT scanners in a single examination and have further improved diagnostic accuracy. Various authors have reported a significant decrease in ambiguous lesions and an improvement in the specificity of PET-CT compared to that of PET alone in the detection of malignant disease [14, 30].
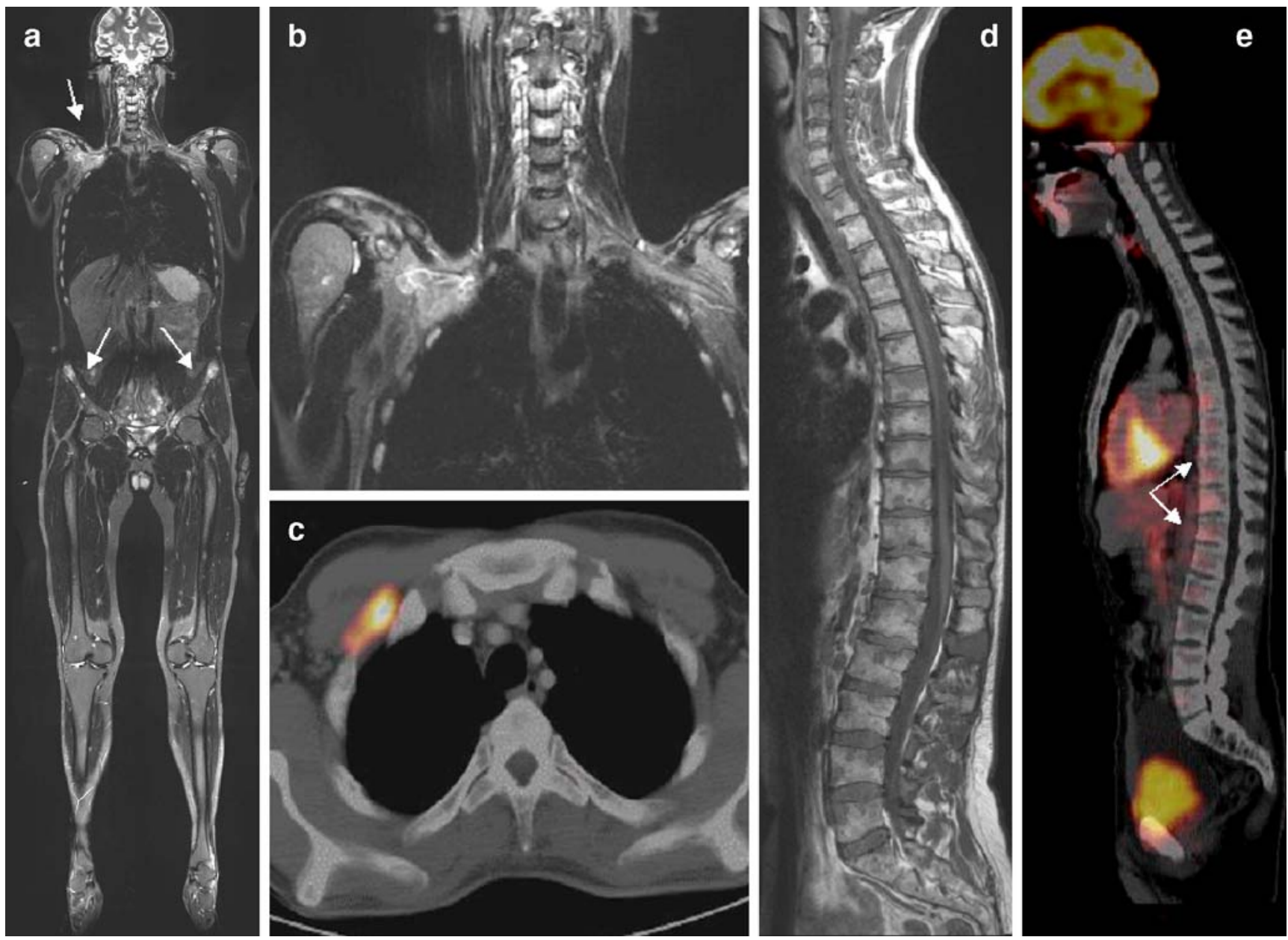

Fig. 2 A 28-year-old man with non-Hodgkin's lymphoma. a Coronal whole-body STIR imaging shows suspect areas with hyperintense signal in the right clavicular region and both iliac bones. b Magnification shows a mass extending from the right clavicular fossa and infiltrating the right apical thoracic cage. c The lesion shows a
Only a few study groups have directly compared the performance of WB-MRI with FDG-PET-CT in the detection of skeletal metastases $[15,31]$. Antoch et al. analyzed the accuracy of both modalities in 98 patients in terms of TNMbased tumor staging. Both imaging procedures revealed a similar diagnostic sensitivity to the detection of distant metastases (WB-MRI 93\%, PET-CT 94\%). Regarding bone metastases, the sensitivity was significantly higher when using WB-MRI (85\%) instead of PET-CT (62\%). Our own observations confirm these data. We examined 41 patients with suspected skeletal metastases with both FDG-PET-CT and WB-MRI using a multi-channel scanner with PAT [31]. In a lesion-by-lesion analysis 102 malignant and 25 benign bone lesions were confirmed by histology or follow-up. WBMRI showed a significantly higher diagnostic accuracy than PET-CT (91\% vs. $78 \%$, Figs. 1, 2). In particular, lesions smaller than $5 \mathrm{~mm}$ were visualized by WB-MRI with a cutoff size of $2 \mathrm{~mm}$ for WB-MRI compared with $5 \mathrm{~mm}$ for PET-CT. Lesions less than double the size of the spatial 
resolution of the PET scanner (usually $6 \mathrm{~mm}$ ) can especially lead to false-negative results. Additionally, 10 bone metastases in distal parts of the body were revealed by WB-MRI due to the larger field of view (the FOV of a routine PET-CT is restricted to the diagnostic spiral $\mathrm{CT}$, usually ranging from the skull base to the proximal femora). However, specificity was higher in PET-CT (PET-CT $80 \%$ vs. WB-MRI 76\%). Here, certainly, the additional metabolic information of PET plays the most important role in reliably discriminating between malignant and benign lesions (e.g., atypical haemangioma) [32].

On the other hand, at least in breast cancer, different patterns of FDG uptake have been reported in osteoblastic, osteolytic or mixed lesions, indicating that sclerotic lesions may be less FDG-avid [33]. The additional morphologic information of PET/CT compared with PET alone is certainly of great value in increasing diagnostic sensitivity. Also, significant improvement in diagnostic accuracy has been reported when $18 \mathrm{~F}$-fluoride is used for PET or PET$\mathrm{CT}$ for the assessment of malignant skeletal disease. 18Ffluoride is a tracer that, similar to ${ }^{99 \mathrm{~m}} \mathrm{TC}$-diphosphonate, specifically adsorbs onto bony surfaces with a predilection for sites of active bone formation [30].

An important indication in bone imaging is lesion monitoring after chemotherapy or radiation therapy. It has to be taken into account that on MRI necrotic bone metastases may remain virtually unchanged in morphology
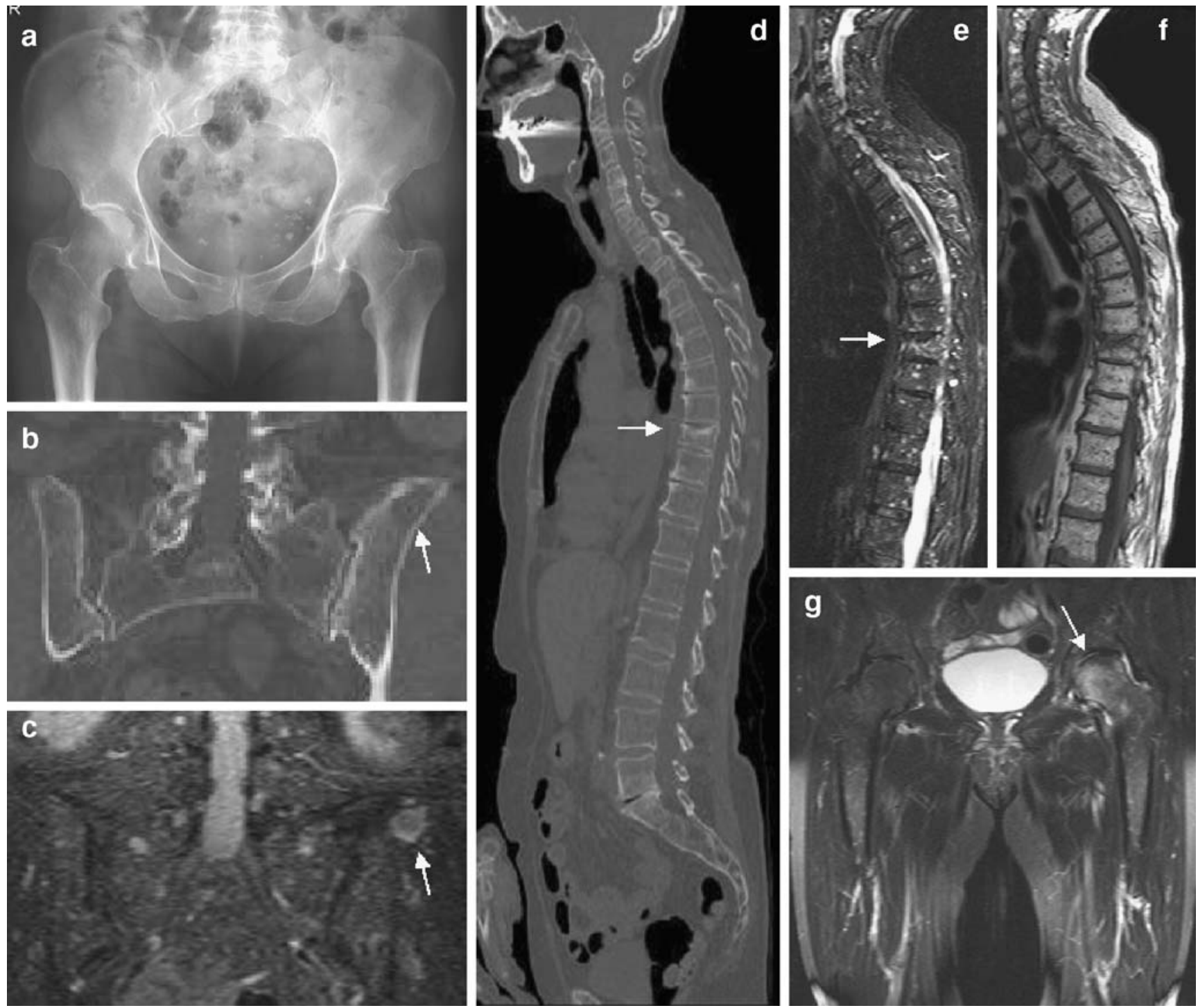

Fig. 3 A 70-year-old man with multiple myeloma. a The radiograph of the pelvis is inconspicuous. b Coronal MS-CT reconstruction of the pelvis in a bone window setting reveals a large area of destruction within the left iliac bone (arrow). c) STIR-WB-MRI confirms focal tumor manifestation within the iliac bone (arrow) and reveals multiple small nodular infiltrations within the sacral bone and pelvis. d MS-CT of the spine shows a compression fracture of Th9. e, f T1-weighted SE- and STIR imaging of the spine reveals diffuse myeloma infiltration of the spine. g Coronal STIR sequences of the pelvis show additional focal infiltration of the left femoral head missed on radiography and MS-CT 
or signal characteristics, which may make evaluation of therapy response difficult. Compared with viable tumors, the contrast enhancement is frequently less pronounced and slower in tumors that have responded to a particular therapy. Reduction in tumor size may be delayed and is not a sensitive sign in the assessment of response. Tumor metabolism, and consequently FDG uptake, is highly susceptible to chemotherapy. On the CT image, osteolytic metastases often demonstrate typical sclerotic transformation. In this setting, future studies are needed to evaluate the sensitivity of FDGPET-CT in assessing the response to chemotherapy and whether sensitivity is reduced in the first days after initiation of therapy. After radiation therapy MRI is particularly helpful, because irradiated lesions are easily distinguishable from new lesions because of the high signal of normal bone marrow on T1-weighted SE images, which is due to conversion of hematopoietic into fatty bone marrow.

\section{Multiple myeloma}

Multiple myeloma is a frequent neoplastic disease affecting the bone marrow. It is characterized by expansive growth of malignant plasma cell clones with consecutive destruction of the bony architecture. Predilection sites are the axial skeleton (spine and pelvis), but also the ribs, the shoulder region, skull, and proximal femurs, stressing the need for total body imaging for adequate assessment of the extent of disease. The bone marrow may either be diffusely infiltrated or there may be focal accumulations of atypical plasma cells (Fig. 3).

In patients with myeloma the basic diagnostic work-up in many institutions includes radiographic examinations of the skull (two planes), the rib cage, the upper arms, the spine (two planes), the pelvis, and the upper legs. This diagnostic approach is still represented in the classic Salmon and Durie staging system of the disease, which includes radiographic, immunohistochemical, and serological factors of the disease, and defines the selection of adequate therapy [34]. However, diagnostic sensitivity of radiography in the detection of myeloma manifestations is rather low and thus allows diagnosis only at advanced stages of the disease when at least $50 \%$ of the bone mineral content has been lost [2]. In particular, a diffuse bone marrow infiltration pattern may cause diagnostic problems as it can easily be misdiagnosed as senile osteoporosis. In a study conducted by Baur et al. $55 \%$ of focal and $59 \%$ of diffuse infiltrations were missed by radiography [35]. With the use of contrast-enhanced sequences and calculation of percentage signal increases the sensitivity of MRI for diffuse myeloma infiltration, especially at earlier stages, can be further increased [36].

Schreiman et al. examined 32 patients with multiple myeloma using single-row CT and radiography [37]. Twelve patients showed osseous affections in both modalities. CT, however, usually demonstrated a more extensive involve- ment and in 6 out of 13 patients only CT detected myeloma involvement despite normal radiographs. Another study group recently proposed FDG-PET-CT as an alternative to radiographic imaging of the whole body [38]. PET-CT detected more lesions in 16 out of 28 patients $(57 \%)$ and 9 of these patients had completely negative radiography.

Ghanem et al. compared WB-STIR-MRI using the conventional radiographic skeletal survey in 54 patients with plasma cell neoplasms [16]. Whole-body MRI correctly revealed bone marrow infiltration in $74 \%$ of patients, while radiography depicted pathological changes in $55 \%$ of patients. Moreover, WB-MRI showed a larger extent of infiltration in $90 \%$ of concordant findings.

In two studies, the diagnostic accuracy of MS-CT and MRI was analyzed. Mahnken et al. compared results of four-detector MS-CT and MRI examination of the spine and pelvis in 18 patients with stage III multiple myeloma [39]. A total number of 325 vertebral bodies were evaluated. In MS-CT 231 vertebral bodies were classified as "affected", compared with only 224 vertebral bodies in MRI. These "false-negative" findings might have been a consequence of response to previous treatment. However, no information about previous therapy was provided in this study. On the other hand, MRI showed 5 affected vertebrae that were considered normal on MS-CT. The false-negatives on MS-CT might be due to early bone marrow infiltration without signs of osseous destruction. Furthermore, the surprisingly high sensitivity of MS-CT in this study may also be caused by pre-selection of a patient group with advanced disease.

In our own study, 30 patients with multiple myeloma were examined with a 1.5 -Tesla multi-channel WB-MRI scanner and a 16- and 64-detector MS-CT scanner [17]. MRI showed superior diagnostic accuracy to MS-CT. In particular, the diagnostic sensitivity of MS-CT was inferior to that of WBMRI (Fig. 3). The high amount of false-negatives on MS-CT may be explained by the fact that early stages of the disease can be visualized on MRI, displaying bone marrow replacement before any destruction of trabecular and cortical bone occurs. False-positive results on MS-CT, on the other hand, may be due to misinterpretation of inhomogeneous osteoporosis as diffuse myeloma infiltration.

Baur et al. were able to show that an extended staging system, including MRI of the spine, has a significant influence on the assessment of prognosis. Using the classic staging system of Durie and Salmon without MRI, 25 out of 77 patients would have been understaged, thus underlining the importance of incorporating the MRI bone marrow status into staging and therapy planning of this disease [40].

Muscle disease

Diagnostic imaging of muscle disease (e.g., polymyositis or muscle dystrophy) represents a challenge as it requires 
high-resolution whole-body coverage of soft tissue structures to adequately assess the pattern, distribution, and extent of the disease. CT, scintigraphy, and ultrasound are not suitable for this purpose as all these modalities lack sufficient soft tissue contrast, spatial resolution or large FOV imaging options. Other diagnostic tests frequently used, like electromyography, are unspecific and muscle biopsies used as the gold standard have been reported to be false-negative in $10-25 \%$ of cases due to sampling errors [41]. MRI, with its precise delineation of fat, muscle, and bone is an ideal candidate for imaging of systemic muscle disease. Moreover, substantial dose exposure in a predom- inantly younger patient cohort commonly affected by muscle diseases can be avoided. Previously, MRI has been used for a targeted assessment of muscle disease [41, 42]. A combination of T1-weighted SE and T2-weighted pulse sequences with fat saturation, such as STIR, are indispensable for assessing increased fat and water content within the muscle tissue respectively (Fig. 4). Contrast enhancement with Gadolinium chelates also adds to diagnostic accuracy, especially when combined with fat suppression. Since various muscle groups and body parts may be involved in an unpredictable distribution, coverage of the whole body is important.
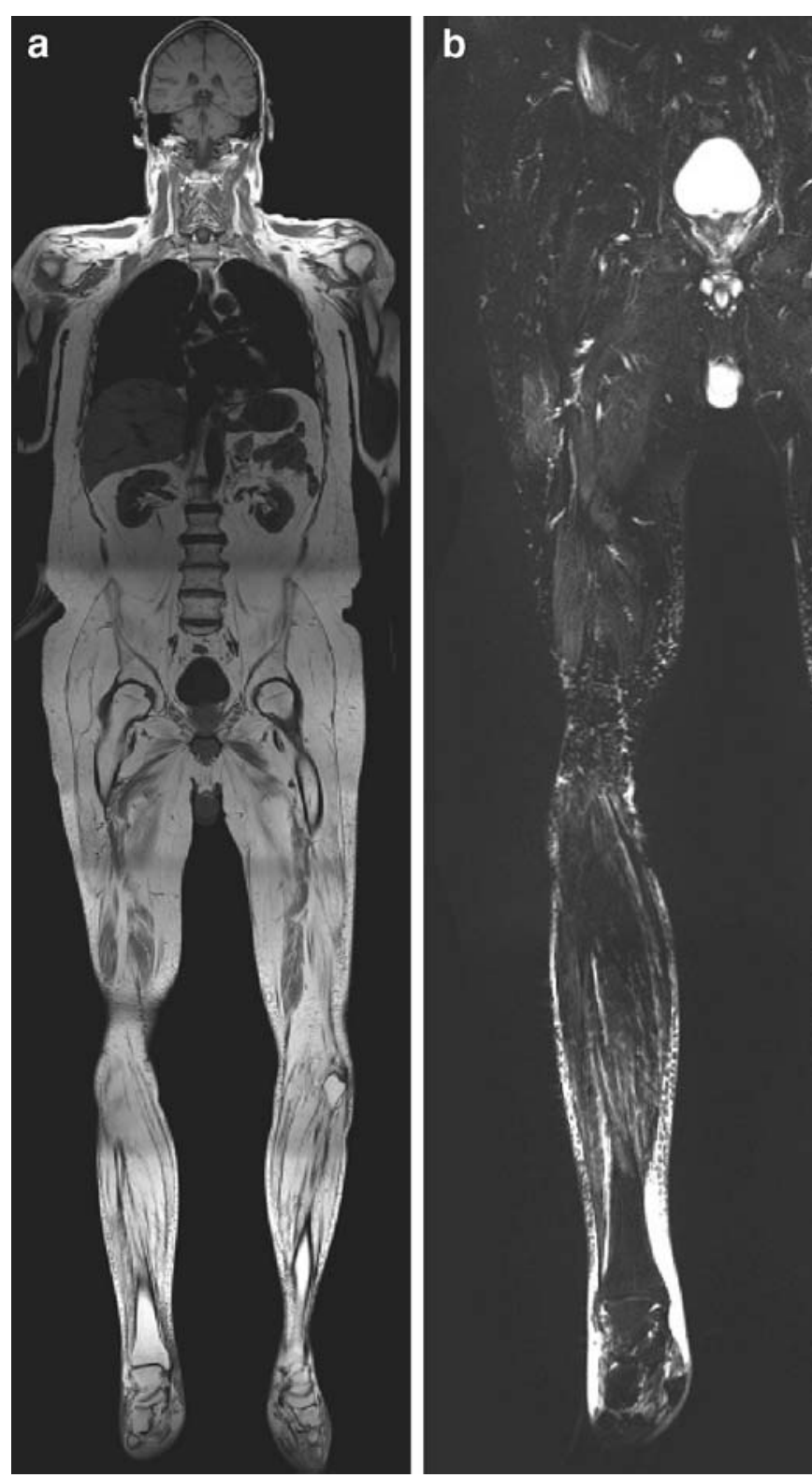

Fig. 4 a Whole-body MRI of a 60-year-old man suffering from severe progressive muscle dystrophy, symmetrically affecting the muscles of the rib cage, abdomen, pelvis, and lower extremities. b WB-STIR shows signs of diffuse reactive edematous processes in the left upper thighs and muscles of the distal lower limbs. c, d T1-
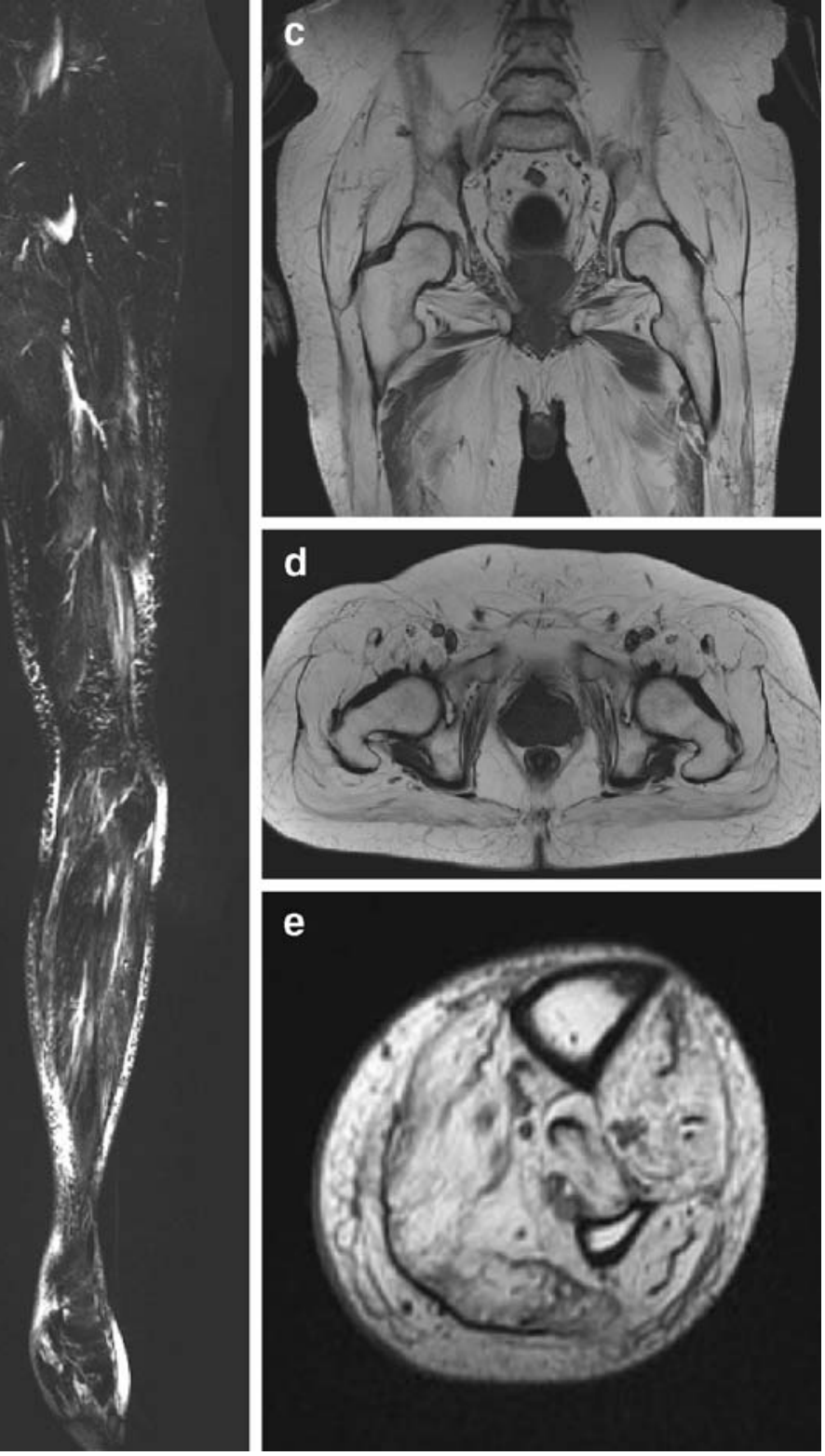

weighted imaging displays fatty tissue replacement affecting most of the muscles of the pelvis. Residual adductor muscle tissue is shown with high contrast due to its hypointense signal. e Progressive muscle dystrophy has also occurred in the lower extremities, affecting all compartments 

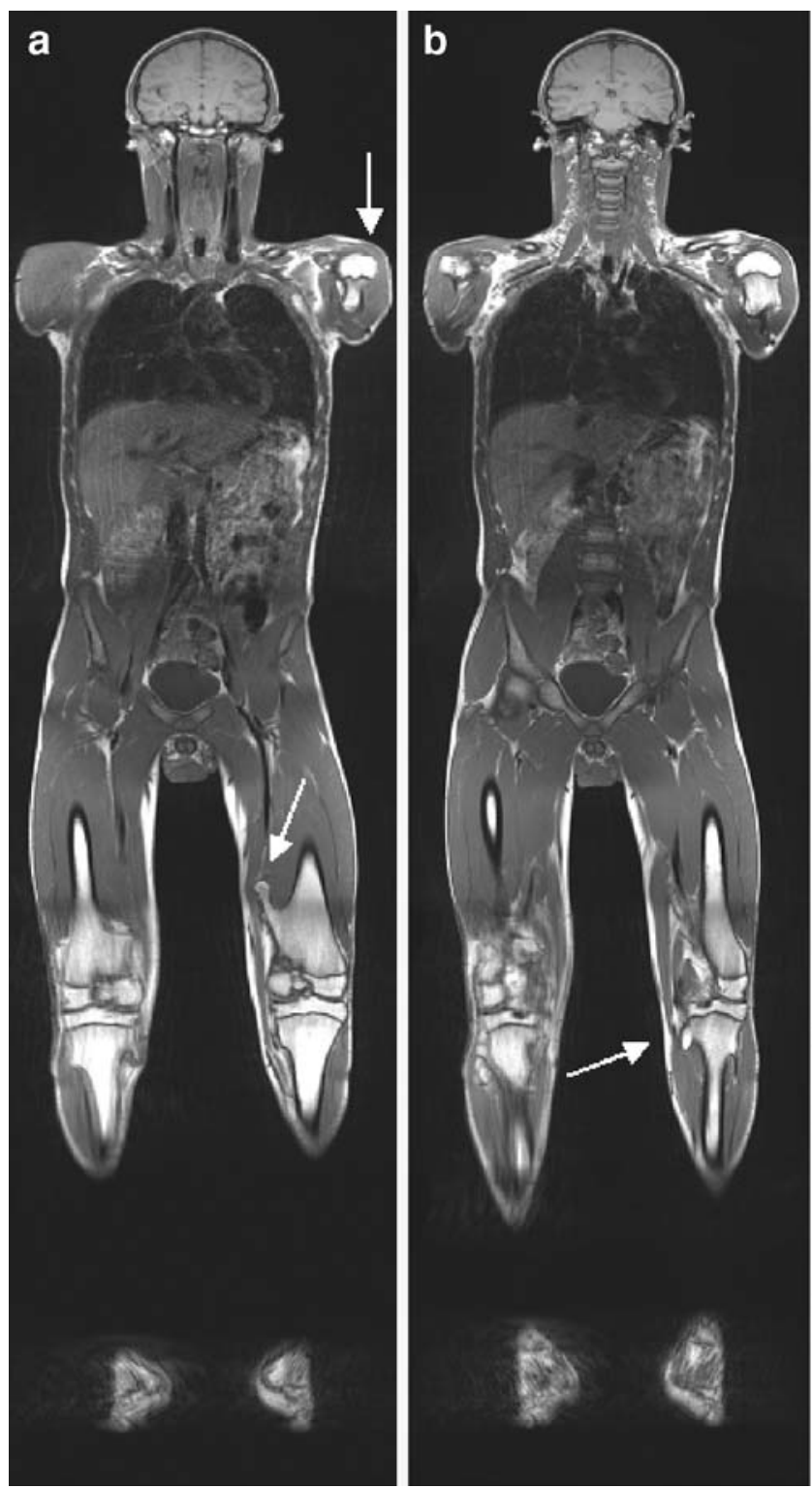

Fig. 5 A 35-year-old man with multiple cartilaginous exostoses. a, b Whole-body MRI shows typical manifestations in the metaphyseal parts of the long bones of the upper and lower extremities. c Enlargement of the left knee joint shows an exostosis at the medial

O'Connell et al. previously described WB-STIR imaging for the diagnosis of patients suffering from polymyositis [18]. With the use of parallel imaging techniques WB-STIR imaging can be performed in under $13 \mathrm{~min}$ at an inplane resolution of $1.3 \times 1.1 \mathrm{~mm}$. With this method, symmetry of muscle involvement and the extent and severity of inflammation can be analyzed, so that an adequate target for biopsy can be identified. During therapy, WB-MRI is particularly helpful in monitoring the disease outcome by visualizing the muscle-to-fat tissue relation or to verify a decrease in inflammation. Also, various drugs, such as steroids, may have side effects that involve the muscles and result in various types of myopathy. Lenk et al. proposed
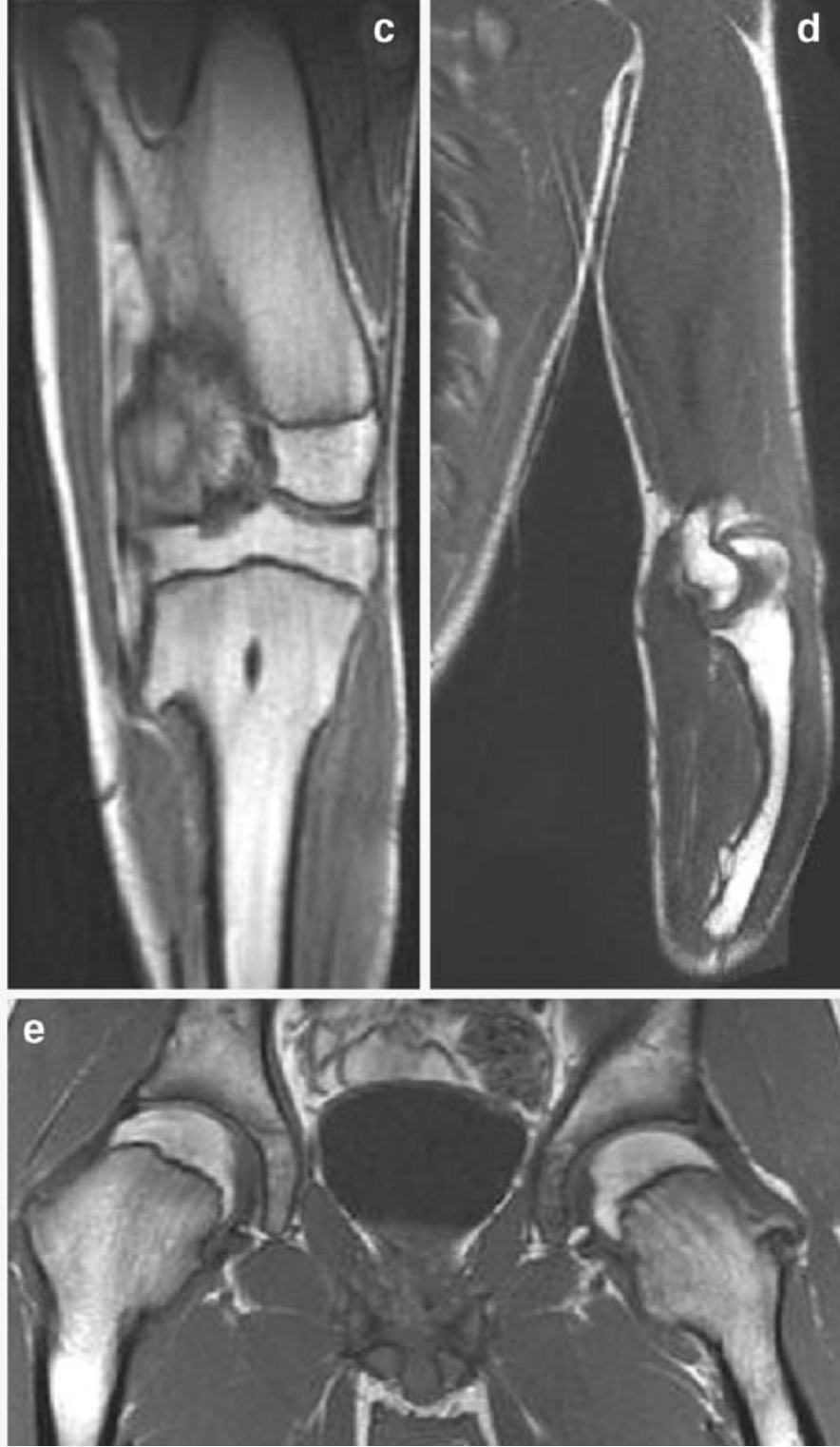

side of the femur and at the proximal tibia. d Deformation of the radius. e Exostoses and deformation of both femoral necks. In summary, no indications of malignant transformation (e.g., widening of the cartilaginous cap) were found in this patient

coronal and axial WB-STIR imaging in combination with coronal T1-weighted SE imaging and sagittal T1-/T2weighted imaging of the spine, as a sensible protocol for systemic muscle disease, resulting in an approximate total scan time of $45 \mathrm{~min}$ [19].

However, assessment of distal parts of the upper extremity may be limited. This is due to positioning of the arms on the pelvis and thighs. If the muscles of the forearm are in the focus of interest, additional examination with dedicated coil systems may be required.

Patients with muscular dystrophy, an X-chromosome recessive disease, suffer from progressive destruction of muscle tissue with subsequent replacement by fatty and 
fibrous tissue. In addition to the morphological information concerning the distribution and extent of replacement of muscle by fatty tissue as provided by WB-MRI, exact quantification of the muscle-to-fat relation is of substantial diagnostic and prognostic importance. In particular, T1weighted axial WB-MRI in combination with CAD (computer aided diagnostics) applications may be useful for the quantification of total body fat distribution. Interesting results have been reported by Brennan et al. who introduced a fast, automated approach to body fat measurement in healthy individuals as a useful alternative to the body mass index [43].

\section{Future applications}

Eustace et al. have proposed WB-MRI for trauma assessment in patients referred for suspected child abuse [44]. For a rapid assessment of acute trauma, especially when multiple organs are affected, MS-CT provides fast and detailed systemic information on potentially life-threatening organ, vessel or bone injury and is the imaging technique of choice. Recently, an accelerated triage MS-CT protocol has been proposed by Koerner et al. for time-effective handling of mass casualty incidents using MS-CT. For a scenario of 15 multiple trauma patients admitted over a period of $2 \mathrm{~h}$ within a mass casualty incident a mean total time in the scanner room of 8.9 min was calculated, including patient preparation and image reconstruction [45].

Finally, there may be potential for WB-MRI in the secondary screening of congenital skeletal diseases predisposing to malignancies, like multiple cartilaginous exostoses or histiocytosis X (Fig. 5). However, only case reports have been published on the potential benefits or therapeutic impact in these specific patient groups.

\section{Conclusion}

Whole-body imaging is increasingly successfully applied in musculoskeletal imaging, especially in the field of systemic malignant diseases affecting the bone and in diseases predisposing to malignant transformation. Whole-body MRI is able to depict bone marrow pathologies with high resolution and excellent soft tissue contrast by demonstrating signal alterations due to changes in its fat, water, and hematopoietic cell components with high resolution and excellent soft tissue contrast. Together with CT or PET-CT and its valuable additional metabolic information, it has great potential in the more comprehensive, more accurate, and earlier diagnosis of musculoskeletal diseases. Although further evaluation of the true potential of whole-body applications is awaited, they are promising tools aiding the more efficient management of patients suffering from systemic malignant or benign diseases of the soft tissue and bone.

\section{References}

1. Rubens RD. Bone metastases: the clinical problem. Eur J Cancer 1998;34: 210-213.

2. Lecouvet FE, Malghem J, Michaux L, et al. Skeletal survey in advanced multiple myeloma: radiographic versus MR imaging survey. Br J Haematol 1999;106: 35-39.

3. Daldrup-Link HE, Franzius C, Link TM, et al. Whole-body MR imaging for detection of bone metastases in children and young adults: comparison with skeletal scintigraphy and FDG PET. AJR Am J Roentgenol 2001;177: 229-236.

4. Imamura F, Kuriyama K, Seto T, et al. Detection of bone marrow metastases of small cell lung cancer with magnetic resonance imaging: early diagnosis before destruction of osseous structure and implications for staging. Lung Cancer 2000;27: 189-197.

5. Krishnamurthy GT, Tubis M, Hiss J, Blahd WH. Distribution pattern of metastatic bone disease. JAMA 1977;237: 837-842.

6. Steinborn M, Heuck AF, Tiling R, Bruegel M, Gauger L, Reiser MF. Whole body bone marrow MRI in patients with metastatic disease to the skeletal system. J Comput Assist Tomogr 1999;23: $123-129$.

7. Lauenstein T, Freudenberg L, Goehde S, et al. Whole body MRI using a rolling table platform for the detection of bone metastases. Eur Radiol 2002;12: 2091-2099.

8. Keupp J, Boernert P, Aldefeld B. Continuously moving table SENSE imaging with exact reconstruction using a 16-coil array. Proc Intl Soc Magn Reson Med 2005;13: 483.

9. Zenge MO, Ladd ME, Vogt FM, Brauck K, Barkhausen J, Quick $\mathrm{HH}$. Whole-body magnetic resonance imaging featuring moving table continuous data acquisition with high-precision position feedback. Magn Reson Med 2005;54: 707-711.

10. Krahe T, Nicolas V, Ring S, Warmuth-Metz M, Koster O. Diagnostic evaluation of full $\mathrm{x}$-ray pictures and computed tomography of bone tumors of the spine. Fortschr Roentgenstr 1989;150: 13-19.

11. Poitout D, Gaujoux G, Lempidakis M, et al. X-ray computed tomography or MRI in the assessment of bone tumor extension. Chirurgie 1991;117(5-6): 488-490.

12. Mulkens TH, Bellinck P, Baeyaert M, et al. Use of an automatic exposure control mechanism for dose optimization in multidetector row CT examinations: clinical evaluation. Radiology 2005;237: 213-223.

13. Antoch G, Vogt FM, Freudenberg LS, et al. Whole-body dualmodality PET-CT and whole-body MRI for tumor staging in oncology. JAMA 2003;290: 3199-3206.

14. Metser U, Lerman H, Blank A, Lievshitz G, Bokstein F, Even-Sapir E. Malignant involvement of the spine: assessment by ${ }^{18}$ FDGPET-CT. J Nucl Med 2004;45: 279-284.

15. Antoch G, Saoudi N, Kuehl H, et al. Accuracy of whole-body dual-modality fluorine-18-2-fluoro-2-deoxy-D-glucose positron emission tomography and computed tomography (FDG-PET/CT) for tumor staging in solid tumors: comparison with CT and PET. J Clin Oncol 2004;22: 4357-4368.

16. Ghanem N, Lohrmann C, Engelhardt M, et al. Whole-body MRI in the detection of bone marrow infiltration in patients with plasma cell neoplasms in comparison to the radiological skeletal survey. Eur Radiol 2006;16: 1005-1014.

17. Buhmann S, Schoenberg S, Becker CR, Lang N, Reiser MF, Baur-Melnyk A. Whole-body imaging approach of patients with 
multiple myeloma: comparing MR imaging with MD-CT. Eur Radiol 2006;16(Suppl 1): B-003.

18. O'Connell MJ, Powell T, Brennan D, Lynch T, McCarthy C, Eustace SJ. Whole-body MR imaging in diagnosis of polymyositis. AJR Am J Roentgenol 2002;179: 967-971.

19. Lenk S, Fischer S, Kotter I, Claussen CD, Schlemmer HP. Possibilities of whole-body MRI for investigating musculoskeletal diseases. Radiologe 2004;44: 844-853.

20. Walker R, Kessar P, Blanchard R, et al. Turbo STIR magnetic resonance imaging as a whole-body screening tool for metastases in patients with breast carcinoma: preliminary clinical experience. J Magn Reson Imaging 2000;11: 343-350.

21. Vanel D, Bittoun J, Tardivon A. MRI of bone metastases. Eur Radiol 1998;8: 1345-1351.

22. Schmidt GP, Baur A, Staebler A, et al. Diffuse bone marrow infiltration of the spine in multiple myeloma: correlation of MRI with histological results. Fortschr Roentgenstr 2005;177: 745-750.

23. Hawighorst H, Libicher M, Knopp MV, Moehler T, Kauffmann GW, Kaick G. Evaluation of angiogenesis and perfusion of bone marrow lesions: role of semiquantitative and quantitative dynamic MRI. J Magn Reson Imaging 1999;10: 286-294.

24. Eustace S, Tello R, DeCarvalho V, et al. A comparison of wholebody turbo STIR MR imaging and planar $99 \mathrm{~m}$ TC-methylene diphosphonate scintigraphy in the examination of patients with suspected skeletal metastases. AJR Am J Roentgenol 1997;169: $1655-1661$.

25. Engelhard K, Hollenbach HP, Wohlfahrt K, Von Imhoff E, Fellner FA. Comparison of whole-body MRI with automated moving table technique and bone scintigraphy for screening for bone metastases in patients with breast cancer. Eur Radiol 2004;14: 99-105.

26. Groves AM, Beadsmoore CJ, Cheow HK, et al. Can 16-detector multislice CT exclude skeletal lesions during tumour staging? Implications for the cancer patient. Eur Radiol 2006;10: 1-8.

27. Baur-Melnyk A, Buhmann S, Wieser A, Reiser MF. MS-CT versus MR imaging: diagnostic sensitivity in the detection of spine metastases. Eur Radiol 2005;15(Suppl 1): B-464.

28. Fogelman I, Cook G, Israel O, Van der Wall H. Positron emission tomography and bone metastases. Sem Nucl Med 2005;35: 135-142.

29. Reske SN, Kotzerke J. FDG-PET for clinical use. Results of the 3rd German interdisciplinary consensus conference. Eur J Nucl Med 2001;28: 1707-1723.

30. Even-Sapir E, Metser U, Flusser G, Zuriel L, Kollender Y, Lerman $\mathrm{H}$. Assessment of malignant skeletal disease: initial experience with $18 \mathrm{~F}$-fluoride PET/CT and comparison between 18F-fluoride PET and 18F-fluoride PET/CT. J Nucl Med 2004;45: 272-278.

31. Schmidt GP, Baur-Melnyk A, Herzog P, et al. High-resolution whole-body magnetic resonance image tumor staging with the use of parallel imaging vs dual-modality positron emission tomogra- phy-computed tomography: experience on 32-channel system. Invest Radiol 2005;40: 743-753.

32. Schmidt GP, Schoenberg SO, Schmid R, et al. Screening for bone metastases: whole-body MRI using a 32-channel system versus dual-modality PET-CT. Eur Radiol 2007;17(4): 939-949.

33. Cook GJ, Houston S, Rubens R, et al. Detection of bone metastases in breast cancer by 18-FDG-PET: differing metabolic activity in osteoblastic and osteolytic lesions. J Clin Oncol 1998;16: 3375-3379.

34. Durie BGM, Salmon SE. A clinical staging system for multiple myeloma: correlation of measured myeloma cell mass with presenting clinical features, response to treatment and survival. Cancer 1975;36: 842-854.

35. Baur A, Stäbler A, Bartl R, Lamerz R, Reiser M. Infiltration pattern of plasmocytoma in MRI. Fortschr Röntgenstr 1996;164: 457-463.

36. Baur A, Staebler A, Bartl R, Lamerz R, Scheidler J, Reiser MF. MRI gadolinium enhancement of bone marrow: age-related changes in normals and diffuse neoplastic infiltration. Skelet Radiol 1997;26: 414-418.

37. Schreiman JS, McLeod RA, Kyle RA, Beabout JW. Multiple myeloma: evaluation by CT. Radiology 1985;154: 483-486.

38. Nanni C, Zamagni E, Farsad M, Castelluci P, Tosi P, Cangini D. Role of $18 \mathrm{~F}-\mathrm{FDG}$ PET/CT in the assessment of bone involvement in newly diagnosed multiple myeloma: preliminary results. Eur J Nucl Med 2006;33: 525-531.

39. Mahnken AH, Wildberger JE, Gehbauer G. Multidetector CT of the spine in multiple myeloma: comparison with MR imaging and radiography. AJR Am J Roentgenol 2002;178: 1429-1436.

40. Baur A, Stäbler A, Nagel D, et al. Magnetic resonance imaging as a supplement for the clinical staging system of Durie and Salmon? Cancer 2002;95: 1334-1345.

41. Farber JM, Buckwalter KA. MR imaging in nonneoplastic muscle disorders of the lower extremity. Radiol Clin North Am 2002;40: 1013-1031.

42. Chan WP, Liu GC. MR imaging of primary skeletal muscle diseases in children. AJR Am J Roentgenol 2002;179: 989-997.

43. Brennan D, Whelan PF, Robinson K, Ghita O, O'Brien JM, Sadleir R. Rapid automated measurement of body fat distribution from whole-body MRI. AJR Am J Roentgenol 2005;185: 418423.

44. Eustace S, Walker RE, Blake M, Yucel EK. Whole-body MR imaging, practical issues, clinical applications and future directions. Magn Reson Clin N Am 1999;40: 1013-1031.

45. Koerner M, Kroetz M, Kanz KG, Pfeiffer KJ, Reiser MF, Linsenmaier U. Development of an accelerated MSCT protocol (triage MSCT) for mass casualty incidents: comparison to singletrauma patients. Eur Radiol 2006;12: 203-209. 